\title{
Quenching and Reactivation of Electroluminescence by Charge Trapping and Detrapping in Si-Implanted Silicon Nitride Thin Film
}

\author{
Zhan Hong Cen, T. P. Chen, Liang Ding, Yang Liu, Zhen Liu, Ming Yang, Student Member, IEEE, \\ Jen It Wong, Student Member, IEEE, W. P. Goh, Fu Rong Zhu, and S. Fung
}

\begin{abstract}
In this brief, quenching of electroluminescence (EL) from Si-implanted silicon nitride (SNR) thin film under a forward bias has been observed. The quenching phenomenon is shown to be due to charge trapping in the defect states involved in the radiative recombination. The composite EL bands have different quenching rates, causing a change in the EL spectrum shape by the $E L$ quenching. Release of the trapped charges by a low-temperature annealing at $120^{\circ} \mathrm{C}$ or an application of a reverse gate bias can partially recover the quenched EL both in the intensity and spectrum shape. The quenching phenomenon poses a serious challenge to the application of Si-implanted SNR thin films in light-emitting devices.
\end{abstract}

Index Terms-Charge trapping, electroluminescence quenching, reactivation, $\mathrm{Si}$-implanted silicon nitride.

\section{INTRODUCTION}

$\mathbf{H}$ IGHLY efficient light-emitting device (LED) compatible with the mainstream $\mathrm{Si}$ technology is an essential component for Si-based optoelectronic integrated circuit. Si-rich silicon nitride (SRN) is a promising candidate material for Si-based LED with advantages such as smaller injection barrier and light emission at the short wavelengths [1]-[3]. Besides tunable photoluminescence [1], strong electroluminescence (EL) in various wavelength ranges, e.g., red light [4], green-yellow light [5], blue light, and even white light [6]-[8], has been obtained from various SRN materials. On the other hand, SRN has prominent memory characteristics and has been widely used as the charge-trapping layer in the

Manuscript received July 2, 2009; revised August 26, 2009. First published October 23, 2009; current version published November 20, 2009. This work was supported by the National Research Foundation of Singapore under Project NRF-G-CRP 2007-01. The work of Y. Liu was supported by NSFC under Project 60806040 . The review of this brief was arranged by Editor L. Lunardi.

Z. H. Cen, T. P. Chen, Z. Liu, M. Yang, and J. I. Wong are with the School of Electrical and Electronic Engineering, Nanyang Technological University, Singapore 639798, Singapore (e-mail: echentp@ntu.edu.sg).

L. Ding was with the School of Electrical and Electronic Engineering, Nanyang Technological University, Singapore 639798, Singapore. He is now with the Institute of Microelectronics, Agency for Science, Technology and Research, Singapore 117685, Singapore.

Y. Liu is with the State Key Laboratory of Electronic Thin Films and Integrated Devices, University of Electronic Science and Technology of China, Chengdu 610054, China.

W. P. Goh and F. R. Zhu are with the Institute of Materials Research and Engineering, Singapore 117602, Singapore.

S. Fung is with the Department of Physics, The University of Hong Kong, Kowloon, Hong Kong.

Color versions of one or more of the figures in this brief are available online at http://ieeexplore.iee.org.

Digital Object Identifier 10.1109/TED.2009.2033009 nonvolatile memory devices [9]. It has been reported that in the case of LEDs based on $\mathrm{SiO}_{2}$ doped with $\mathrm{Si}, \mathrm{Ge}$, or $\mathrm{Er}$, charge trapping in the luminescent active layer influences the LED performance and can lead to structural breakdown and EL efficiency degradation [10]-[12]. However, the influence of charge trapping on the EL from SRN is seldom reported, although it is important to the LED operation. In this brief, stability of the EL from Si-implanted SNR thin film has been investigated. The EL quenches during the device operation under a forward bias, and the EL bands exhibit different quenching rates. The EL quenching is attributed to charge trapping in the Si-implanted thin film. The observed EL quenching can be partially reactivated by a low-temperature heat treatment or an application of a reverse bias, which can cause a partial release of the trapped charges.

\section{EXPERIMENTAL DETAILS}

An SNR thin film with the designed thickness of $30 \mathrm{~nm}$ was deposited on the (100)-oriented $p$-type Si substrate by lowpressure chemical-vapor-deposition technique. Multiple Si ion implantations into the SNR thin film were carried out, followed by thermal annealing at $1100{ }^{\circ} \mathrm{C}$ in $\mathrm{N}_{2}$ ambient for $1 \mathrm{~h}$. The multiple Si ion implantations include the following: the first implantation with the dose of $4 \times 10^{16}$ atoms $/ \mathrm{cm}^{2}$ at the energy of $25 \mathrm{keV}$, the second implantation with $8 \times 10^{15}$ atoms $/ \mathrm{cm}^{2}$ at $8 \mathrm{keV}$, and the third implantation with $3 \times 10^{15}$ atoms $/ \mathrm{cm}^{2}$ at $2 \mathrm{keV}$. A nearly uniform distribution of implanted $\mathrm{Si}$ in the SNR thin film with the excess Si concentration of $\sim 1.2 \times$ $10^{22} \mathrm{~cm}^{-3}$ was obtained. The annealed Si-implanted SNR thin film was characterized by X-ray photoelectron spectroscopy (XPS) using a Kratos AXIS spectrometer with monochromatic Al $K \alpha(1486.71 \mathrm{eV}) \mathrm{X}$-ray radiation. The XPS spectra were calibrated with the $\mathrm{C} 1 \mathrm{~s}$ spectra from the sample surface due to contamination. Fig. 1 shows the XPS spectra of Si 2p core level that was taken at the depth of $\sim 20 \mathrm{~nm}$ below the thin-film surface. The Si 2 p spectra can be decomposed into two Gaussian peaks related to the $\mathrm{Si}^{4+}$ and $\mathrm{Si}^{0}$ states corresponding to the two chemical structures of $\mathrm{Si}_{3} \mathrm{~N}_{4}$ and $\mathrm{Si}$, respectively [13]. The $\mathrm{Si}^{0}$ component confirms the existence of Si nanocrystals in the annealed Si-implanted SNR thin film.

The metal-insulator-semiconductor (MIS)-like LED structures were fabricated by using a 130-nm indium tin oxide (ITO) layer and a 200-nm aluminum (Al) layer to form the top and bottom electrodes, respectively. The diameter of the circular 


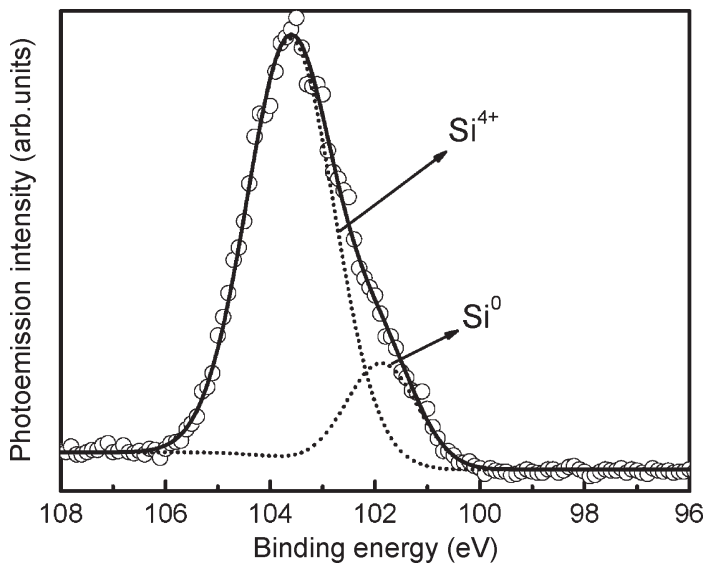

Fig. 1. Decomposition of the Si 2p XPS peak taken at the depth of $\sim 20 \mathrm{~nm}$ in the annealed Si-implanted SNR thin film.

ITO electrode is $1.2 \mathrm{~mm}$. For the capacitance-voltage $(C-V)$ measurement, the structures with Al top electrode instead of the ITO electrode were also prepared. EL spectra were measured at room temperature over the photon-energy range of $1.37-4.13 \mathrm{eV}(300-900 \mathrm{~nm})$ under constant-current injection with a negative gate voltage (i.e., forward bias). A Keithley 2400 sourcemeter was used to supply a constant current for the EL measurement. $C-V$ measurements were performed with a Keithley 4200 semiconductor characterization system at $1 \mathrm{MHz}$, and the voltage range is set to be from -11 to $0 \mathrm{~V}$ such that there is no significant charging during the $C-V$ measurements.

\section{Results AND Discussions}

The EL spectra under the constant gate current of $-8 \mathrm{~mA}$ (the corresponding voltage is about $-15 \mathrm{~V}$ ) at the beginning and after the operation time of 16 min are shown in Fig. 2(a). The EL spectra can be deconvoluted into four Gaussian-shape bands [8], as shown in Fig. 2(a). The EL spectra consist of two major bands located at 2.2 and $3.0 \mathrm{eV}$, which are denoted as green-yellow band and violet band, respectively. Two minor EL bands, including an ultraviolet (UV) band at $3.8 \mathrm{eV}$ and a near-infrared (IR) band at $1.46 \mathrm{eV}$, can be obtained as well. The violet band can be attributed to the radiative transition from the defect state related to the silicon dangling bonds $\left(\equiv \mathrm{Si}^{0}\right)$ located at $3.1 \mathrm{eV}$ above the valence-band maximum (VBM) to the bonding state $(\sigma)$ of the $\equiv \mathrm{Si}-\mathrm{Si} \equiv$ unit that is close to the valence band [6], [14], [15]. While the green-yellow band could be due to the transition between the $\equiv \mathrm{Si}^{0}$ state and the state related to the nitrogen dangling bonds $\left(=\mathrm{N}^{-}\right)$in the valenceband tail, which is located at $0.8 \mathrm{eV}$ above the VBM [14], [15]. Meanwhile, the radiative recombination of electrons injected into the conduction band of SNR and holes in the valenceband tail produces the UV band emission [8]. Moreover, the near-IR band is attributed to the radiative transition in the $\mathrm{Si}$ nanocrystals that can be formed in the SNR thin film during the postimplantation high-temperature annealing. The radiative recombination processes for the EL bands are schematically shown in Fig. 2(b).

As shown in Fig. 2(a), after the operation of $16 \mathrm{~min}$, the EL quenches, i.e., the EL intensity decreases greatly. The EL-
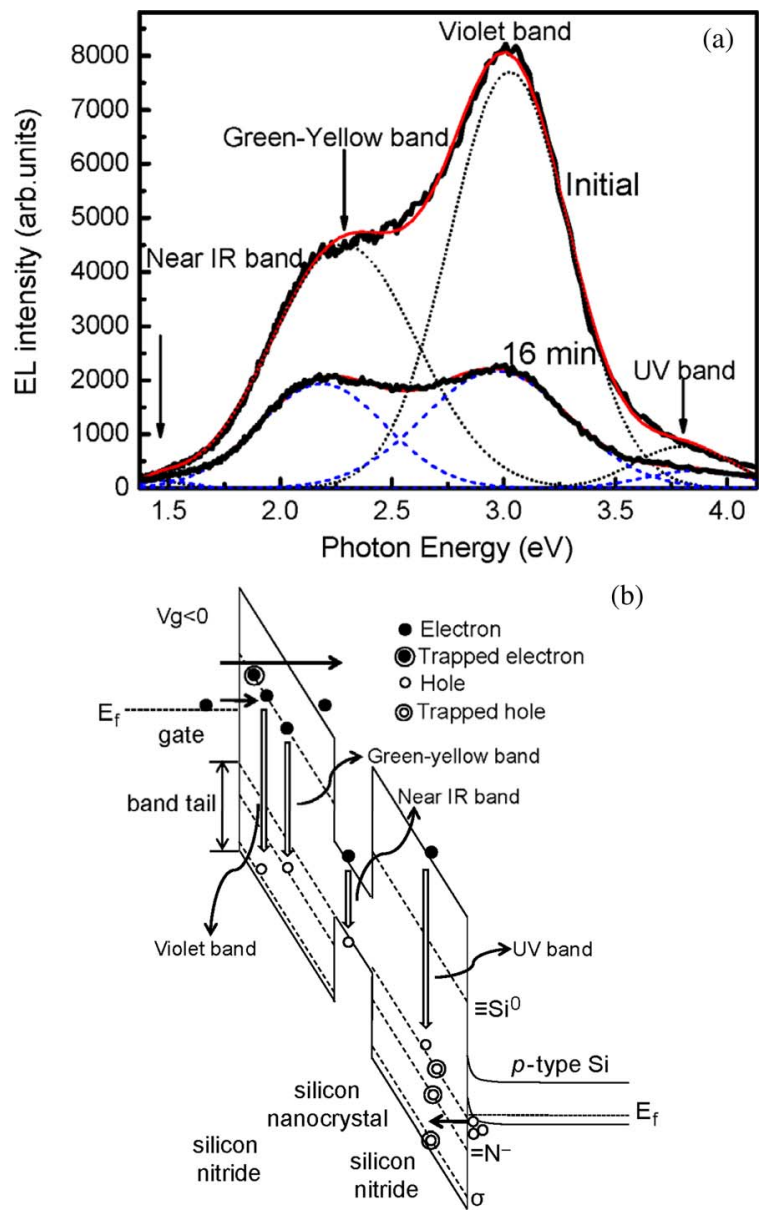

Fig. 2. (a) EL spectra under a constant current of $-8 \mathrm{~mA}(\sim-15 \mathrm{~V})$ obtained from either a fresh device or after the device operation for $16 \mathrm{~min}$. The deconvolution of the EL spectra into four EL bands is also shown in (a). (b) Schematic band diagram of the LED structure under forward bias. Carrier transport, charge trapping, defect states, and Si nanocrystal in the nitride film and various recombination processes are also illustrated in the diagram.

quenching phenomenon is also observed for constant-voltage injection. During the EL-quenching process, no significant shifts of the EL bands can be observed, indicating that the light-emission mechanisms governing the EL bands do not change during the quenching. Fig. 3 shows the evolution of the normalized intensity of the four EL bands with the operation time. The two major EL bands, i.e., the violet band and the green-yellow band, both exhibit decreases in intensity following an exponential decay, but the quenching rate of the violet band is larger than that of the green-yellow band. As a consequence, the shape of EL spectrum changes with the operation time, as shown in Fig. 2(a). Similar to the two major EL bands, the UV band quenches significantly also, with a quenching rate close to that of the green-yellow band. However, the quenching of the other minor EL band (i.e., the near-IR band) is not very significant. As the EL bands correspond to different recombination routes for the injected electrons and holes, the different quenching rates of the EL bands could be related to the respective luminescence centers involved in the light-emission processes.

On the other hand, the $C-V$ measurement shows that the carrier injection leads to the charge trapping in the Si-implanted 


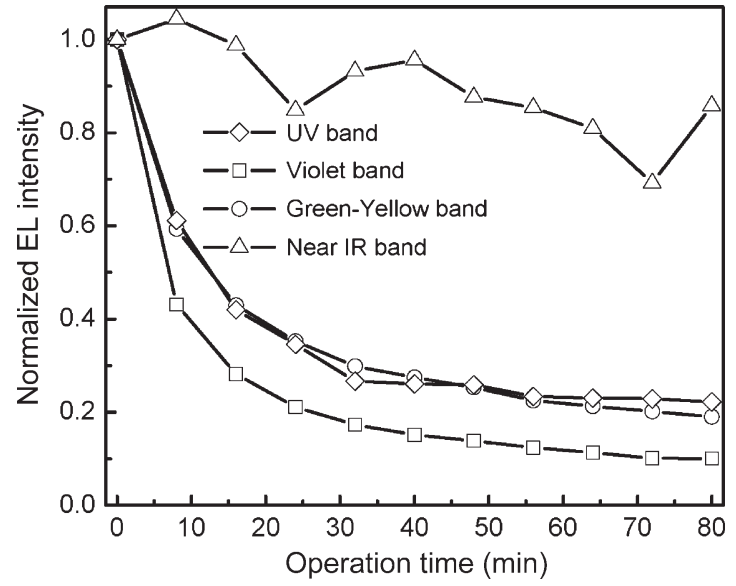

Fig. 3. Evolution of the normalized intensity of the four EL bands with operation time.

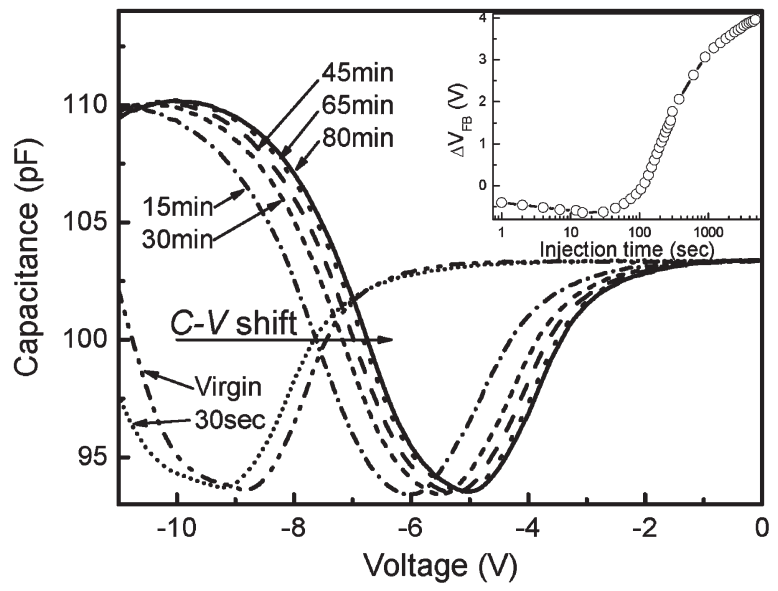

Fig. 4. Shifts in the $C-V$ characteristic caused by the carrier injection at $-15 \mathrm{~V}$ for different injection time. The inset shows $\Delta V_{\mathrm{FB}}$ as a function of the injection time.

SNR thin film. Fig. 4 shows the shifts in the $C-V$ characteristic of the Si-implanted film caused by the carrier injection at $-15 \mathrm{~V}$ for different injection time. A shift in the $C-V$ curve can be translated into a flatband voltage shift $\left(\Delta V_{\mathrm{FB}}\right)$ of the MIS structure, which is an indicator of charge trapping in the insulator [16][17]. The inset of Fig. 4 shows the $\Delta V_{\mathrm{FB}}$ as a function of the injection time. For a short injection time $(\lesssim 110 \mathrm{~s})$, the $\Delta V_{\mathrm{FB}}$ is negative, indicating a net positive charge (i.e., hole) trapping; for injection time longer than $\sim 110 \mathrm{~s}$, the $\Delta V_{\mathrm{FB}}$ is positive, indicating a net negative charge (i.e., electron) trapping. Under the forward-bias condition, electrons are injected from the gate electrode while holes are injected from the $p$-type Si substrate. Some of both injected electrons and holes are trapped in the Si-implanted SNR layer, and the net charge trapping revealed by the $C-V$ shift could be either positive or negative depending on the injection time. The injected electrons and holes could be trapped in the defect states in the Si-implanted SNR layer. As discussed later, the charge trapping in the defect states should affect the light emission, since the defect states are involved in the radiative recombination processes.

A quenched EL can be reactivated quickly by a lowtemperature annealing, as shown in Fig. 5(a). As shown in
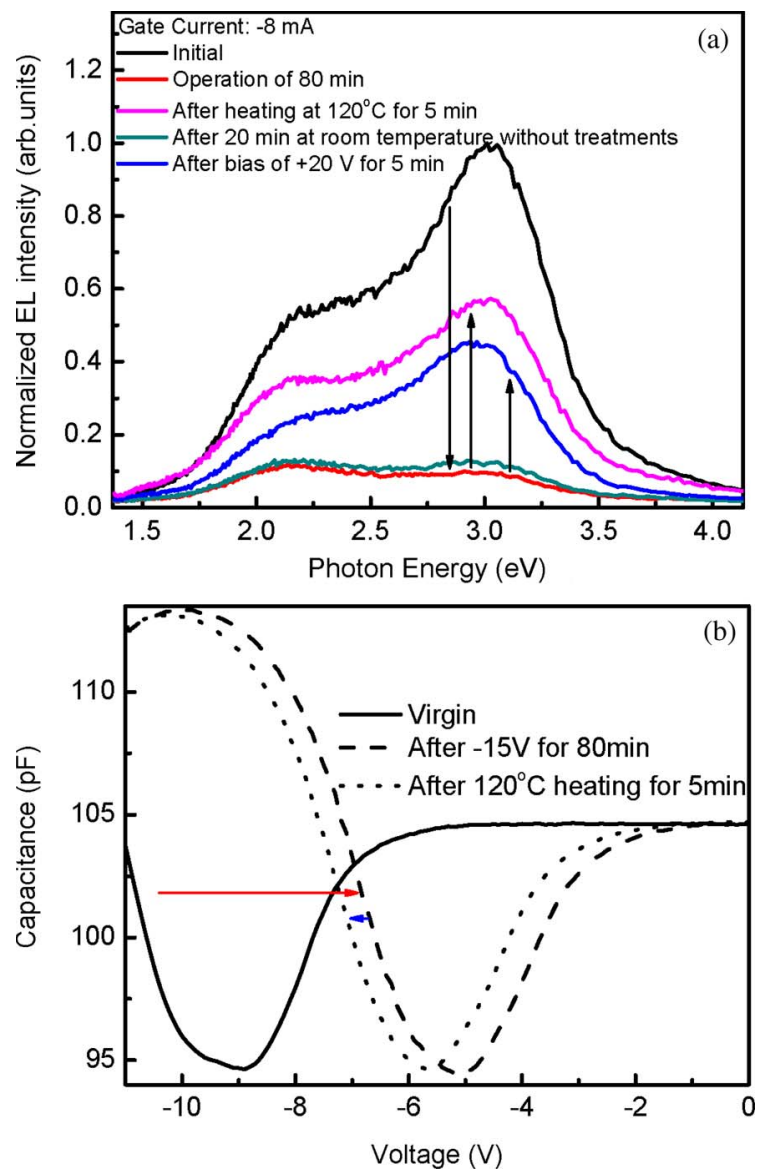

Fig. 5. (a) Reactivation of the quenched EL by either an annealing at $120^{\circ} \mathrm{C}$ for $5 \mathrm{~min}$ or an application of $+20 \mathrm{~V}$ for $5 \mathrm{~min}$. A slight restoration of the quenched EL after $20 \mathrm{~min}$ without any annealing treatments at room temperature is also shown in (a). (b) Partial recovery of the shifted $C-V$ characteristic by the annealing at $120^{\circ} \mathrm{C}$ for $5 \mathrm{~min}$.

the figure, the quenched EL for the operation time of $80 \mathrm{~min}$ under the gate current of $-8 \mathrm{~mA}$ can be partially recovered in intensity by the annealing at $120{ }^{\circ} \mathrm{C}$ for $5 \mathrm{~min}$. Moreover, the annealing also leads to a recovery of the shape of the EL spectrum. Similar to the EL-quenching process, the violet band shows more sensitivity to the annealing than the other three bands in the EL-reactivation process. As a result of the annealing, the intensity of the quenched violet band, green-yellow band, and UV band increases by a factor of $\sim 5$, $\sim 3$, and $\sim 2.5$, respectively; while the near-IR band shows only a slight increase in its intensity. The suppressed violet band in the quenched EL spectrum now becomes the dominant band again after the annealing (note that the violet band is the dominant one in the initial EL spectrum). The EL reactivation by annealing is related to the release of some of the trapped charges in the Si-implanted thin film during the heat treatment, which can be evidenced by the $C-V$ measurement. As shown in Fig. 5(b), the annealing leads to a partial recovery in the $C-V$ characteristic, i.e., a shift toward the virgin one. The $C-V$ shift clearly demonstrates the partial release of the trapped charges. Even without any annealing treatments to the device, a slight restoration of the quenched EL can be also observed at room temperature after a certain time as a result of the release of some of the trapped charges. For example, a small increase in 
the quenched EL intensity was observed after switching off the LED for 20 min, as shown in Fig. 5(a).

EL reactivation similar to the low-temperature annealing can be achieved by an application of a reverse (positive) gate bias as well. The effect of the application of $+20 \mathrm{~V}$ for $5 \mathrm{~min}$ on the quenched EL is shown in Fig. 5(a). By the reversed bias, the quenched EL is partially recovered with the intensity of the violet and green-yellow major EL bands increased by a factor of 4.5 and 2.5, respectively. Meanwhile, the intensity of the two minor EL bands is also increased. Since the correlation between EL and charge trapping has been established earlier, the EL reactivation can be explained by the charge release under the reverse bias. Under the positive bias, some of the electrons that are trapped near the gate during the device operation are released. The release of the trapped electrons will enhance the light emission as discussed earlier, but the electron release cannot be revealed by the $C-V$ measurement as the measurement is not sensitive to a change in the charge trapping near the gate. On the other hand, the hole trapping close to the Si substrate is reduced by the electron injection from the substrate inversion layer under the positive bias. This is confirmed by the $C-V$ measurement that shows a $C-V$ shift to more positive voltage after the application of the positive bias. The release of some of the electrons trapped near the gate and holes trapped near the substrate causes the partial recovery of the quenched EL.

EL quenching in MIS-like LEDs has been reported to be strongly related to the charge trapping in the luminescent layer [10]-[12]. For example, under the hot electron impact excitation and high electric field injection $(8-10 \mathrm{MeV} / \mathrm{cm})$, the charge trapping can cause transformation of the luminescence centers to optically inactive centers and permanently destroy to the EL and even breakdown to the LED structure [11], [12]. However, the EL quenching under a relatively low electric field (about $5 \mathrm{MeV} / \mathrm{cm}$ ) in this brief can be quickly reactivated through release of the trapped charges by a low-temperature annealing or an application of a reverse bias. The origin of the EL changes by charge trapping/detrapping in the Si-implanted SNR thin film may be related to the defect states existing in the thin film, which provide channels for electron-hole radiative recombination. On the other hand, the defect states related to $\equiv \mathrm{Si}^{0}$ can act as the electron traps and the states in valenceband tail such as $\sigma$ and $=\mathrm{N}^{-}$states as the hole traps during the carrier injection [15], [18], as shown in Fig. 2(b). When the $\equiv \mathrm{Si}^{0}$ states are occupied by the trapped electrons during the LED operation, the electron trapping near the gate will lead to a reduction in electric field for electron injection from the ITO gate [10]. Similarly, the hole trapping in the valence-band tail states near the interface between the Si-implanted film and Si substrate can reduce the electric field for the hole injection from the substrate [10]. As the electron and hole injections into the luminescent centers are reduced or the injected electrons and holes are unbalanced for radiative recombination, the EL is quenched consequently, even though the constant-current injection can be sustained by other carrier transport mechanisms such as the Fowler-Nordheim injection of electrons into the SNR conduction band. When the trapped charges are released by the excitation of external thermal energy or electric field, the electron injection from the gate and hole injection from the substrate into the luminescent centers can be recovered, and thus, the EL is reactivated. In addition, due to the relatively shallow position of the $\sigma$ state, as shown in Fig. 2(b), the hole trapping/detrapping in the $\sigma$ state should be easier than in the $=\mathrm{N}^{-}$state or other valence-band tail states. Therefore, the violet band involving the $\sigma$ state should be more sensitive to the quenching and reactivation processes than the green-yellow band involving the $=\mathrm{N}^{-}$state and the UV band involving other valence-band tail states. As a consequence, the quenching of the violet band is faster than both the green-yellow and UV bands, and so does the reactivation process. On the other hand, being different from the earlier three bands involving the defect states, the near-IR band, which is attributed to the radiative recombination in the $\mathrm{Si}$ nanocrystals, is much less sensitive to the EL quenching and reactivation processes.

\section{CONCLUSION}

In conclusion, EL quenching in the Si-implanted SNR thin film has been studied. The EL quenching is due to the charge trapping in the defect states existing in the thin film involved in the radiative recombination of the injected electrons and holes. The quenched EL can be reactivated by the release of the trapped charges with a low-temperature annealing or an application of a reverse bias. The composite EL bands have different quenching/reactivation rates, which is due to the different abilities of charge trapping/detrapping in the respective defects of the different bands.

\section{REFERENCES}

[1] N.-M. Park, C.-J. Choi, T.-Y. Seong, and S.-J. Park, "Quantum confinement in amorphous silicon quantum dots embedded in silicon nitride," Phys. Rev. Lett., vol. 86, no. 7, pp. 1355-1357, Feb. 2001.

[2] L. Dal Negro, J. H. Yi, L. C. Kimerling, S. Hamel, A. Williamson, and G. Galli, "Light emission from silicon-rich nitride nanostructures," Appl. Phys. Lett., vol. 88, no. 18, p. 183 103, May 2006.

[3] C.-H. Lin, W.-Y. Uen, S.-M. Lan, Y.-C. Huang, S.-M. Liao, Z.-Y. Li, T.-N. Yang, C.-T. Ku, M.-C. Chen, and Y.-H. Huang, "Luminescence mechanisms of silicon-rich nitride films fabricated by atmospheric pressure chemical vapor deposition in $\mathrm{N}_{2}$ and $\mathrm{H}_{2}$ atmospheres," J. Appl. Phys., vol. 105, no. 5, p. 053 107, Mar. 2009.

[4] J. Warga, R. Li, S. N. Basu, and L. Dal Negro, "Electroluminescence from silicon-rich nitride/silicon superlattice structures," Appl. Phys. Lett., vol. 93 , no. 15, p. 151116 , Oct. 2008.

[5] R. Huang, K. J. Chen, P. G. Han, H. P. Dong, X. Wang, D. Y. Chen, W. Li, J. Xu, Z. Y. Ma, and X. F. Huang, "Strong green-yellow electroluminescence from oxidized amorphous silicon nitride light-emitting devices," Appl. Phys. Lett., vol. 90, no. 9, p. 093 515, Mar. 2007.

[6] L.-Y. Chen, W.-H. Chen, and F. C. N. Hong, "Visible electroluminescence from silicon nanocrystals embedded in amorphous silicon nitride matrix," Appl. Phys. Lett., vol. 86, no. 19, p. 193 506, May 2005.

[7] Z. W. Pei, Y. R. Chang, and H. L. Hwang, "White electroluminescence from hydrogenated amorphous SiNx thin films," Appl. Phys. Lett., vol. 80, no. 16, pp. 2839-2841, Apr. 2002.

[8] Z. H. Cen, T. P. Chen, L. Ding, Y. Liu, J. I. Wong, M. Yang, Z. Liu, W. P. Goh, F. R. Zhu, and S. Fung, "Strong violet and green-yellow electroluminescence from silicon nitride thin films multiply implanted with Si ions," Appl. Phys. Lett., vol. 94, no. 4, p. 041 102, Jan. 2009.

[9] S. Choi, H. Yang, M. Chang, S. Baek, H. Hwang, S. Jeon, J. Kim, and C. Kim, "Memory characteristics of silicon nitride with silicon nanocrystals as a charge trapping layer of nonvolatile memory devices," Appl. Phys. Lett., vol. 86, no. 25, p. 251 901, Jun. 2005.

[10] Y. Liu, T. P. Chen, L. Ding, M. Yang, J. I. Wong, C. Y. Ng, S. F. Yu, Z. X. Li, C. Yuen, F. R. Zhu, M. C. Tan, and S. Fung, "Influence of charge trapping on electroluminescence from Si-nanocrystal light emitting structure," J. Appl. Phys., vol. 101, no. 10, p. 104 306, May 2007. 

Tyagulskii, V. S. Lysenko, S. Prucnal, T. Gebel, and L. Rebohle, "Quenching of electroluminescence and charge trapping in high-efficiency Ge-implanted MOS light-emitting silicon diodes," Appl. Phys. B, Lasers Opt., vol. 87, no. 1, pp. 129-134, Mar. 2007. W. Skorupa, "Reactivation of damaged rare earth luminescence centers in ion-implanted metal-oxide-silicon light emitting devices," Appl. Phys. B, Lasers Opt., vol. 91, no. 1, pp. 123-126, Apr. 2008.

[13] Y. Liu, T. P. Chen, Y. Q. Fu, M. S. Tse, J. H. Hsieh, P. F. Ho, and Y. C. Liu, "A study on Si nanocrystal formation in Si-implanted $\mathrm{SiO} 2$ films by X-ray photoelectron spectroscopy," J. Phys. D, Appl. Phys., vol. 36, no. 19, pp. L97-L100, Oct. 2003.

[14] S. V. Deshpande, E. Gulari, S. W. Brown, and S. C. Rand, "Optical properties of silicon nitride films deposited by hot filament chemical vapor deposition," J. Appl. Phys., vol. 77, no. 12, pp. 6534-6541, Jun. 1995.

[15] J. Robertson and M. J. Powell, "Gap states in silicon nitride," Appl. Phys. Lett., vol. 44, no. 4, pp. 415-417, Feb. 1984.

[16] C. N. Berglund, "Surface states at steam-grown silicon-silicon dioxide interfaces," IEEE Trans. Electron Devices, vol. ED-13, no. 10, pp. 701-

[17] E. H. Nicollian and J. R. Brews, MOS (Metal Oxide Semiconductor) Physics and Technology. New York: Wiley, 1982.

[18] W. L. Warren, J. Kanicki, J. Robertson, E. H. Poindexter, and P. J. McWhorter, "Electron paramagnetic resonance investigation of charge trapping centers in amorphous silicon nitride films," J. Appl. Phys., vol. 74, no. 6, pp. 4034-4046, Sep. 1993.

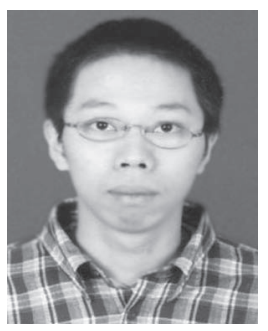

Zhan Hong Cen received the B.Sc. degree in physics and the M.Sc. degree in physics (microelectronics) from Nanjing University, Nanjing, China, in 2003 and 2006, respectively. He is currently working toward the Ph.D. degree in the School of Electrical and Electronic Engineering, Nanyang Technological University, Singapore, Singapore.

His current research interests include optoelectronic properties and the applications of semiconduc-
[11] A. N. Nazarov, I. N. Osiyuk, J. M. Sun, R. A. Yankov, W. Skorupa, I. P.

[12] S. Prucnal, L. Rebohle, A. N. Nazarov, I. N. Osiyuk, I. P. Tjagulskii, and 705, Oct. 1966. tor nanocrystals.

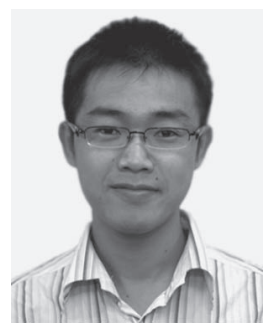

Liang Ding was born in China in 1982. He received the B.Sc. degree in physics (optical and optoelectronic physics) from Huazhong University of Science and Technology, Wuhan, China, in 2004 and the $\mathrm{Ph} . \mathrm{D}$. degree from the Division of Microelectronics, School of Electrical and Electronic Engineering, Nanyang Technological University, Singapore, Singapore, in 2009.

Since October 2008, he has been with the Institute of Microelectronics, Agency for Science, Technology and Research, Singapore, where he is currently a Senior Research Engineer. His current research interest involves Si photonic devices based on SOI platform, and optoelectronic properties of semiconductor nanocrystal materials and the device applications.

Dr. Ding was the recipient of the prestigious Singapore Millennium Foundation Ph.D. Fellowship Award in 2007 and the Chinese Government Award for Outstanding Self-financed Student Abroad in 2008.

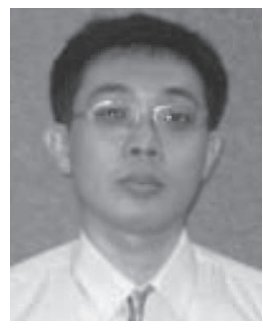

Yang Liu received the B.Sc. degree in microelectronics from Jilin University, Changchun, China, in 1998 and the Ph.D. degree from Nanyang Technological University, Singapore, Singapore, in 2005.

From May 2005 to July 2006, he was a Research Fellow with Nanyang Technological University. Since 2008 , he has been with the University of Electronic Science and Technology of China, Chengdu, China, where he is currently a Professor with the School of Microelectronics. His current research focus on nanocrystals/nanoparticles and their applications in electronic devices (Flash memory device, resistive memory device, power device, etc.) and optoelectronic devices (Si-based light-emission devices, optoelectronic memory), and Si-based optical ICs. Since 2002, he has authored/coauthored more than 60 peer-reviewed journal papers and 20 conference papers.

Dr. Liu was the recipient of the prestigious two-year Singapore Millennium Foundation Fellowship Award in 2006.

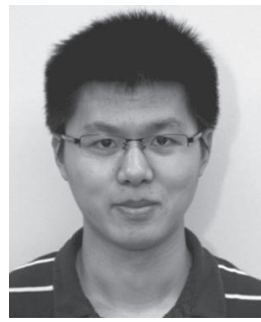

Zhen Liu was born in Changsha, China, in November 1983. He received the B.Eng. degree in microelectronics from Nanyang Technological University, Singapore, Singapore, in 2006, where he is currently working toward the Ph.D. degree in the School of Electrical and Electronic Engineering.

His research interest involves the synthesis and characterizations of metal nanoparticles, targeting on the electrical, optical, and optoelectronic applications.

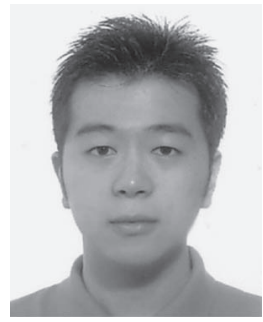

Ming Yang (S'06) was born in Xiamen, China, in 1982. He received the B.Eng. degree (with first class honors) in microelectronics from Nanyang Technological University, Singapore, Singapore, in 2005, where he is currently working toward the Ph.D. degree in the School of Electrical and Electronic Engineering.

His research interest involves the electrical and optoelectronic properties of nanoscale materials and their device applications.

December 1996, he was with Chartered Semiconductor Manufacturing, Ltd. He was a Senior Scientist with PSB Singapore for two and a half years, before he joined Nanyang Technological University, Singapore, in February 2000. He is currently an Associate Professor with the Division of Microelectronics, School of Electrical and Electronic Engineering, Nanyang Technological University. He is the author or coauthor of 170 peer-reviewed journal papers, more than 80 conference proceeding papers, and one book chapter. He is the holder of two granted and one pending U.S. patents. His current research interests include nanoscale CMOS devices and reliability physics, semiconductor and metal nanocrystals/nanoparticles and their applications in nanoelectronic devices (memories and single/few-electron devices) and photonic/optoelectronic devices (light emitters, Plasmon waveguides, optical interconnects), Si optoelectronic integrated circuits for chip-to-chip and system-to-system communication, and flexible/printing electronics.

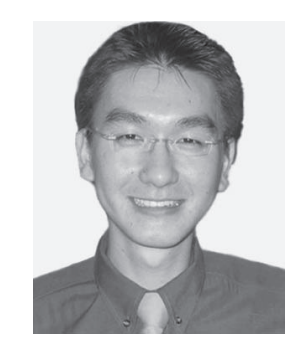

Jen It Wong ( $\mathrm{S}^{\prime} 03$ ) was born in Johor Bahru, Malaysia, in 1982. He received the B.Eng. degree in microelectronics from the School of Electrical and Electronic Engineering, Nanyang Technological University, Singapore, Singapore, in 2005, where he is currently working toward the Ph.D. degree.

His research focuses on studies of semiconductor nanocrystals with particular interest on their application for optoelectronic device and memory device. 


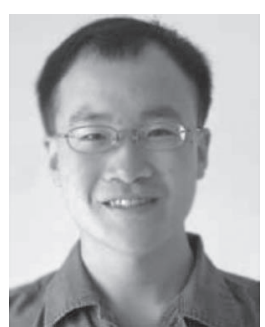

W. P. Goh received the B.Sc. degree in applied science (applied physics) from the Royal Melbourne Institute of Technology University, Melbourne, Australia, in 2004 and the M.Sc. degree in material physics and nanotechnology from Linköping University, Linköping, Sweden, in 2006

$\mathrm{He}$ is currently with the Institute of Materials Research and Engineering, Singapore, Singapore.

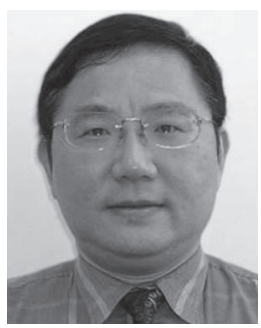

Fu Rong Zhu received the Ph.D. degree from Charles Darwin University, Darwin, Australia, in 1993.

From 1993 to 1995, he did his postdoctorate with the Department of Electrical and Electronic Engineering, Kyoto University, Kyoto, Japan. From 1995 to 1997, he was a Research Fellow with Murdoch University, Perth, Australia. Since 1997, he has been with the Institute of Materials Research and Engineering, Singapore, Singapore, where he is currently a Program Manager. His current research includes OLEDs, organic photovoltaics, and thin-film-materials-oriented research.

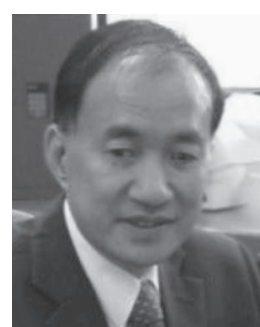

Physics, U.K.
S. Fung was born in Hong Kong in 1950. He received the B.A. degree in physics (with first class honors), the Ph.D. degree in semiconductor physics, and the D.Sc. degree from the University of Oxford, Oxford, U.K., in 1977, 1980, and 1996, respectively.

$\mathrm{He}$ is currently a Professor with the Department of Physics, The University of Hong Kong, Kowloon, Hong Kong. His major research interests include nanomaterials, wide bandgap semiconductor, and positron physics.

Dr. Fung is a Fellow of the Institute of 